\title{
Topological Superconductivity Could be a Twist Away
}

\author{
Theoretical models of twisted graphene bilayers explain a recently detected superconducting \\ phase, which might harbor topological states that are desired for quantum computing.
}

\section{by Balázs Dóra*}

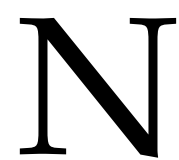

o one would dispute that graphene is an excit-
ing material, or that its discoverers were worthy
of a Nobel prize [1]. But if you're a lover of
materials in which electrons are strongly correlated, graphene's single layer of carbon atoms hasn't been much of a draw. That impression changed earlier this year, when two exceptional experiments were reported on "twisted graphene." This atypical form of graphene consists of a pair of closely spaced graphene layers rotated so that their carbon atoms are slightly out of alignment (Fig. 1). The first experiment showed that a small twist angle caused the conduction electrons to form a Mott insulator [2], in which the charges are "frozen" onto distinct lattice sites by strong electron-electron interactions. The second experiment revealed that the insulator can be transformed into a superconductor by using various applied voltages to control the electron density in the graphene layers [3]. This observed transition from Mott insulator to superconductor suggests that graphene exhibits an unconventional form of superconductivity, as found in high-temperature (high- $T_{\mathcal{c}}$ ) superconductors.

Inspired by these findings, theorists are now bringing their toolkit of strongly correlated electron models to twisted graphene, and they are making predictions that may, in turn, inspire the experimentalists. A model from Cenke $\mathrm{Xu}$ of the University of California, Santa Barbara, and Leon Balents of the Kavli Institute for Theoretical Physics, Santa Barbara [4], predicts that superconductivity in twisted graphene is not only unconventional but also topological, a property of interest for certain quantum computation proposals. Unconventional superconductivity (though not the topological kind) is also predicted by two other twisted graphene models from Noah Yuan and Liang Fu of the Massachusetts Institute of Technology, Cambridge [5], and from Hoi Chun Po of Harvard University [6].

\footnotetext{
* Department of Theoretical Physics and MTA-BME Lendület Topology and Correlation Research Group, Budapest University of Technology and Economics, Budapest, Hungary
}

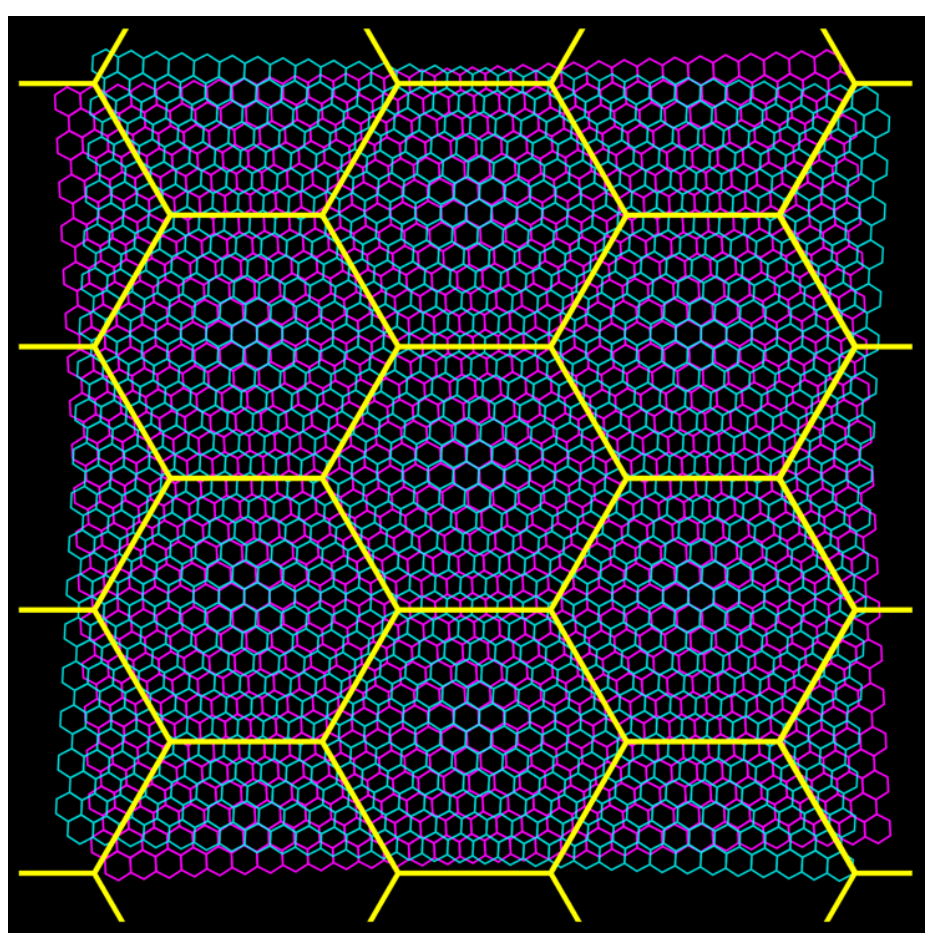

Figure 1: Twisted bilayer graphene consists of two closely spaced graphene sheets, with one sheet slightly rotated with respect to the other. The twist creates a superlattice for the conduction electrons whose periodicity is much greater than the spacing between the carbon atoms. For the twist shown here, the conduction electrons can be treated as though they move in a hexagonal superlattice (yellow lines) or a triangular superlattice (the centers of the yellow hexagons.) (APS/Alan Stonebraker)

"Conventional" superconductivity in simple metals like mercury can be explained with the Bardeen-CooperSchrieffer (BCS) theory, which says that electrons pair up thanks to an attractive interaction mediated by phonons. But most would agree that the same theory cannot describe more complex materials in which superconductivity occurs at much higher temperatures [7]. For example, BCS predicts a superconducting gap that is isotropic in the electron momentum, a situation described as "s-wave," 
whereas a lower symmetry, " $d$-wave," gap is found in many high-temperature (high- $T_{c}$ ) superconductors. If graphene is indeed a model system for a high- $T_{\mathcal{C}}$ superconductor, as some of its features suggest, then studying it could help researchers develop a proper high- $T_{C}$ theory.

The new models provide a framework for such studies [4-6]. They aren't, however, starting completely from scratch. A 2011 theoretical study of twisted bilayer graphene, which treated the electrons as noninteracting, had explored the effect of twist angle on the material's electron energy bands [8]. For certain twist angles, some regions of the carbon atoms in the two layers will line up better than others, leading to a periodic modulation of the electron density. This "moiré pattern" (Fig. 1) has a much longer periodicity than the spacing between the carbon atoms and acts as a superlattice for the conduction electrons. The 2011 paper predicted that at certain "magic" twist angles, such as $1.1^{\circ}$, the bands associated with two degenerate superlattices would become flat when there are two electrons for each site in the superlattice-a condition known as half filling. This turns out to be an ideal situation for strongly correlated electron behavior to kick in: Electrons in flat bands have little kinetic energy, so interactions among the charges dominate their behavior.

The new models pick up this story by assuming the electrons lie in flat bands and that they interact with one another through a repulsive force. The model by $\mathrm{Xu}$ and Balents relies on the observation that the density of the flat-band electrons is highest in regions where the two honeycomb layers have almost perfect overlap. For certain twist angles, these so-called AA regions (Fig. 1) form a triangular lattice (for more details, see Ref. [5]). Xu and Balents therefore argue that the simplest description of the bilayer's behavior treats its electrons as though they are hopping around on an effective triangular lattice. Each electron in their model can be in one of four "flavors": It can have its spin up or spin down, and it can be in one of two possible "valley" orbitals-a spin-like degree of freedom particular to materials with graphene's honeycomb-lattice structure. The electrons also repel each other, but only when they are on the same site. With these ingredients, $\mathrm{Xu}$ and Balents calculate the material's ground state for various electron densities in the limit of strong repulsive interactions.

Their model predicts a Mott insulating state at half filling, in agreement with experiments [2,3]. At higher electron densities, the coupling between the spin and valley orbital degrees of freedom favors two possible superconducting ground states, both of which correspond to unconventional superconductivity because they have a gap with $d$-wave symmetry. Which of these states appears in real materials will have to be determined with experiments. But it's of note that both predicted states are also topological. One superconducting phase, for example, possesses eight so-called Majorana modes that are confined to the edge of the material. These low-energy excitations are of interest for quantum computing because they can potentially store quantum information with less sensitivity to environmental disturbances than other quantum bit systems. The researchers have explained how the two exotic ground states could be distinguished in a transport measurement [4].

The models from Yuan and Fu [5] and from Po et al. [6] predict some similar features. But the devil is in the details. These papers focus on a pair of flat bands, which originate from a more complicated, and probably more realistic, description of the electron kinetic and interaction energies. Specifically, in their models, the electrons hop around in an effective honeycomb lattice. This results in the electrons having eight, instead of four, possible flavors. Not surprisingly, such models support more intricate phases whose relevance for twisted bilayer graphene will have to be determined by future experiments.

This new family of models gives us a lot to explore. The most interesting avenue is the topological nature of the superconductivity predicted by $\mathrm{Xu}$ and Balents. If Majorana edge states exist, as predicted, then they could serve as a solid platform for topological quantum computation and technologies [9]. At a minimum, we could learn to manipulate the states in a simple solid-state system. Another fascinating thing to study is superconductivity in proximity to a Mott insulator. Combining the right models with experiments in which important parameters can be tuned may help us analyze this scenario in enough detail to resolve the mystery of high-temperature superconductivity, which has endured for 30 years. It still remains to be seen whether twisted bilayer graphene can provide the answers to these questions. But regardless, aficionados of strong electron correlations are paying attention.

This research is published in Physical Review Letters, Physical Review B, and on the arXiv.

\section{REFERENCES}

[1] A. H. Castro Neto et al., "The electronic properties of graphene," Rev. Mod. Phys. 81, 109 (2009).

[2] Y. Cao et al., "Correlated insulator behaviour at half-filling in magic-angle graphene superlattices," Nature 556, 80 (2018).

[3] Y. Cao et al., "Unconventional superconductivity in magic-angle graphene superlattices," Nature 556, 43 (2018).

[4] C. Xu and L. Balents, "Topological superconductivity in twisted multilayer graphene," Phys. Rev. Lett. 121, 087001 (2018).

[5] N. F. Q. Yuan and L. Fu, "Model for the metal-insulator transition in graphene superlattices and beyond," Phys. Rev. B 98, 045103 (2018).

[6] H. C. Po, L. Zou, A. Vishwanath, and T. Senthil, "Origin of Mott insulating behavior and superconductivity in twisted bilayer graphene," arXiv:1803.09742.

[7] B. Keimer, S. A. Kivelson, M. R. Norman, S. Uchida, and J. Zaanen, "From quantum matter to high-temperature superconductivity in copper oxides," Nature 518, 179 (2015). 


\section{Physīcs}

[8] R. Bistritzer and A. H. MacDonald, "Moiré bands in twisted double-layer graphene," Proc. Natl. Acad. Sci. U.S.A. 108, 12233 (2011).

[9] M. A. Nielsen and I. L. Chuang, Quantum computation and quantum information (Cambridge University Press, Cambridge, 2010).

10.1103/Physics.11.84 